\title{
(-)-Epigallocatechin gallate attenuates the induction of HSP27 stimulated by sphingosine 1-phosphate via suppression of phosphatidylinositol 3-kinase/Akt pathway in osteoblasts
}

\author{
HIDEO NATSUME $^{1,2}$, SEIJI ADACHI ${ }^{1}$, SHINJI TAKAI ${ }^{1}$, HARUHIKO TOKUDA $^{1,3}$, \\ RIE MATSUSHIMA-NISHIWAKI ${ }^{1}$, CHIHO MINAMITANI ${ }^{1,2}$, JUNICHI YAMAUCHI $^{1}$, \\ KANEFUSA KATO $^{4}$, JUN MIZUTANI ${ }^{2}$, OSAMU KOZAWA ${ }^{1}$ and TAKANOBU OTSUKA ${ }^{2}$ \\ ${ }^{1}$ Department of Pharmacology, Gifu University Graduate School of Medicine, \\ Gifu 501-1194; ${ }^{2}$ Department of Orthopedic Surgery, Nagoya City University Medical Sciences, \\ Nagoya 467-8601; ${ }^{3}$ Department of Clinical Laboratory, National Hospital for Geriatric Medicine, \\ National Center for Geriatrics and Gerontology, Obu 474-8511; ${ }^{4}$ Department of Biochemistry, \\ Institute for Developmental Research, Aichi Human Service Center, Kasugai 486-0392, Japan
}

Received February 16, 2009; Accepted April 21, 2009

DOI: 10.3892/ijmm_00000223

\begin{abstract}
We previously reported that sphingosine 1-phosphate stimulates the induction of heat shock protein 27 (HSP27) through p38 mitogen-activated protein (MAP) kinase and phosphatidylinositol 3-kinase/Akt in osteoblast-like MC3T3-E1 cells. In the present study, we investigated whether (-)-epigallocatechin gallate (EGCG), the major polyphenol found in green tea, affects the induction of HSP27 in these cells and its mechanism, since it was previously reported that catechin, including EGCG, suppresses bone resorption. EGCG significantly reduced the induction of HSP27 stimulated by sphingosine 1-phosphate in a dose-dependent manner between 10 and $30 \mu \mathrm{M}$. Immunofluorescence microscopy studies revealed that sphingosine 1-phosphate certainly stimulated the induction of HSP27 in the cytosol of these cells, and that EGCG clearly suppressed its induction. However, sphingosine 1-phosphate-stimulated phosphorylation of p38 MAP kinase or MAPKAP-2 was not affected by EGCG. By contrast, EGCG markedly suppressed the phosphorylations of both Akt and glycogen synthase kinase-3ß stimulated by sphingosine 1-phosphate. These results strongly suggest that EGCG suppresses the induction of HSP27 stimulated by sphingosine 1-phosphate via attenuation of, not the p38 MAP kinase pathway, but of the phosphatidylinositol 3-kinase/Akt pathway in osteoblasts.
\end{abstract}

Correspondence to: Dr Osamu Kozawa, Department of Pharmacology, Gifu University Graduate School of Medicine, Gifu 501-1194, Japan

E-mail address: okozawa@gifu-u.ac.jp

Key words: epigallocatechin gallate, sphingosine 1-phosphate, heat shock protein 27 , protein kinase, osteoblast

\section{Introduction}

It is generally recognized that heat shock proteins (HSPs) are stimulated in cells in response to biological stress such as heat and chemical stress (1). HSPs are classified into highmolecular-weight HSPs such as HSP90 and 70, and lowmolecular-weight HSPs based on apparent molecular sizes. Low-molecular-weight HSPs with molecular masses from 10 to $30 \mathrm{kDa}$, such as HSP27, $\alpha \mathrm{B}$-crystallin and HSP20, share high homology in amino acid sequences ' $\alpha$-crystallin domain' $(2,3)$. Though the functions of low-molecular-weight HSPs are known less than those of high-molecular-weight HSPs, it is generally believed that they have chaperoning functions like the high-molecular-weight HSPs $(2,3)$. It is well recognized that HSP27 activity is regulated by post-translational modification such as phosphorylation $(4,5)$. Under unstimulated conditions, HSP27 exists as a high molecular weight aggregated form. It is rapidly dissociated as a result of phosphorylation $(6,7)$. The phosphorylation-elicited dissociation from the aggregated form correlates with the loss of molecular chaperone activity $(6,7)$.

Bone metabolism is regulated by two functional cells, osteoblasts and osteoclasts, responsible for bone formation and resorption, respectively (8). The maintenance of bone structures and bone remodeling result from the coupling process, bone resorption by activated osteoclasts with subsequent deposition of new matrix by osteoblasts. In osteoblasts, it was shown that down-regulation of proliferation is accompanied by a transient increase of the HSP27 mRNA expression (9). In addition, heat-stimulated induction of HSP27 is reportedly facilitated by estrogen (10). These findings lead us to speculate that HSP27 takes part in the coordination of osteoblast functions. However, the exact roles of HSP27 in osteoblasts remain to be clarified.

Sphingosine 1-phosphate is a metabolite of sphingomyelin in plasma membrane. It is generally recognized that sphingomyelin is catalyzed by sphingomyelinase, resulting in the 
formation of ceramide, which is subsequently metabolized to sphingosine and sphingosine 1-phosphate. Accumulating evidence indicates that sphingosine 1-phosphate plays an important role in essential cellular functions such as proliferation, differentiation and migration (11-13). In the process of bone remodeling, it was proposed that sphingosine 1-phosphate produced by osteoclasts plays a crucial role in the recruitment of osteoprogenitors to sites of bone resorption (14). As for osteoblasts, it was reported that sphingosine 1-phosphate prevents apoptosis via phosphatidylinositol 3 kinase (PI3-kinase) in primary calvaria rat osteoblasts and human osteosarcoma SaOS-2 cells (15). In our previous studies $(16,17)$, we showed that sphingosine 1-phosphate stimulates the induction of HSP27 via p38 mitogen-activated protein (MAP) kinase and PI3-kinase/Akt in osteoblast-like MC3T3-E1 cells.

It is well known that compounds in foods such as vegetables and fruits have beneficial properties for human beings. Among them, flavonoids reportedly show antioxidative, antiproliferative and proapoptotic effects $(18,19)$. Catechin is one of the major flavonoids, which is present in various species of plants such as tea (18). In bone metabolism, it was reported that catechin suppresses bone resorption (20). As for osteoblasts, it was shown that catechin stimulates alkaline phosphatase activity, a mature osteoblast phenotype and reduces the production of bone-resorptive cytokines, such as tumor necrosis factor- $\alpha$ (TNF- $\alpha$ ) and interleukin-6 (IL-6) in osteoblast-like MC3T3-E1 cells (21). On the other hand, we reported that (-)-epigallocatechin gallate (EGCG) enhances vascular endothelial growth factor (VEGF) synthesis stimulated by prostaglandin $\mathrm{F}_{2_{\alpha}}$ via upregulating stressactivated protein kinase/c-Jun N-terminal kinase (SAPK/JNK) activation in MC3T3-E1 cells (22). In addition, we recently reported that EGCG inhibits prostaglandin $\mathrm{D}_{2}$-stimulated HSP27 induction via suppression of the p44/p42 MAP kinase pathway in these cells (23). But so far, the precise mechanism underlying the effects of EGCG on bone metabolism is not yet clarified.

In the present study, we investigated the effect of EGCG on the induction of HSP27 stimulated by sphingosine 1-phosphate in osteoblast-like MC3T3-E1 cells. Here we show that EGCG suppresses the induction of HSP27 stimulated by sphingosine 1-phosphate via inhibition of PI3-kinase/Akt pathway but not p38 MAP kinase pathway in these cells.

\section{Materials and methods}

Materials. Sphingosine 1-phosphate was purchased from Sigma Chemical Co. (St. Louis, MO). EGCG was obtained from Calbiochem-Novabiochem (La Jolla, CA). Antibodies for phospho-specific p38 MAP kinase, p38 MAP kinase, phospho-specific MAP kinase-activated protein kinase-2 (MAPKAP-2), MAPKAP-2, phospho-specific Akt, Akt, phospho-specific glycogen synthase kinase-3ß (GSK-3ß) and GSK-3ß were purchased from Cell Signaling Technology, Inc. (Beverly, MA). HSP27 antibodies for Western blot analysis were obtained from R\&D Systems, Inc. (Minneapolis, MN). Antibodies for glyceraldehyde 3 phosphate dehydrogenase (GAPDH) and HSP27 for immunofluorescence microscopy studies were obtained from Santa Cruz Biotechnology, Inc.
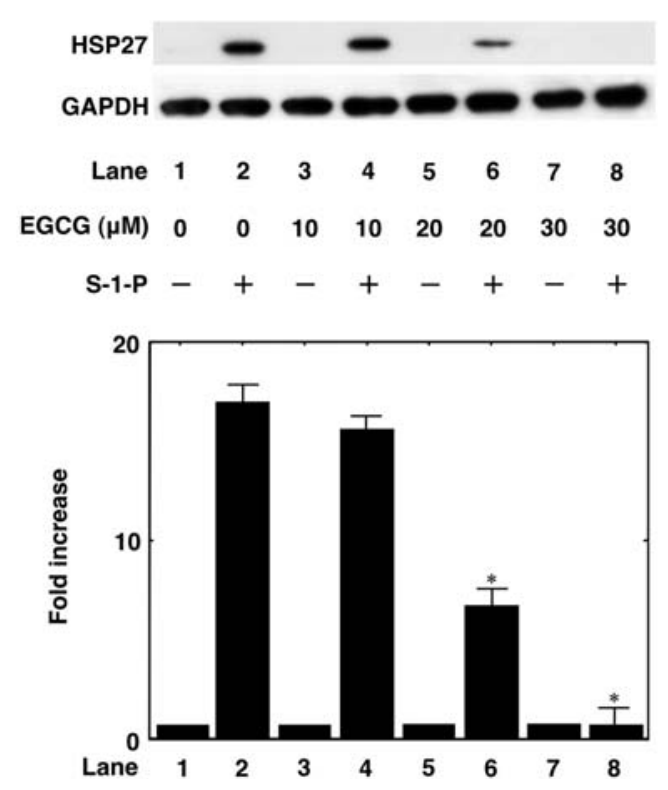

Figure 1. Effect of EGCG on the sphingosine 1-phosphate-stimulated HSP27 induction in MC3T3-E1 cells. The cultured cells were pretreated with various doses of EGCG for $60 \mathrm{~min}$, and then stimulated with $30 \mu \mathrm{M}$ of sphingosine 1-phosphate for $9 \mathrm{~h}$. The extracts of cells were subjected to SDS-PAGE with subsequent Western blotting with antibodies against HSP27 or GAPDH. The lower histogram shows quantitative representations of the levels of sphingosine 1-phosphate-stimulated HSP27 obtained from laser densitometric analysis of three independent experiments. Each value represents the mean \pm SEM of triplicate determinations. Similar results were obtained with two additional and different cell preparations. ${ }^{*} \mathrm{p}<0.05$, compared to the value of sphingosine 1-phosphate alone.

(Santa Cruz, CA). Alexa Fluor $488^{\circledR}$ conjugated goat antirabbit antibodies and Alexa Fluor $555^{\circledR}$ phalloidin were obtained from Invitrogen Corporation, Inc. (Carlsbad, CA). An ECL Western blotting detection system was obtained from Amersham Japan (Tokyo, Japan). Other materials and chemicals were obtained from commercial sources. Sphingosine 1-phosphate was dissolved in dimethyl sulfoxide. The maximum concentration of dimethyl sulfoxide was $0.1 \%$, which did not affect the results.

Cell culture. Cloned osteoblast-like MC3T3-E1 cells derived from newborn mouse calvaria (24) were maintained as previously described (25). Briefly, the cells were cultured in $\alpha$-minimum essential medium ( $\alpha$-MEM) containing $10 \%$ fetal calf serum (FCS) at $37^{\circ} \mathrm{C}$ in a humidified atmosphere of $5 \%$ $\mathrm{CO}_{2}$ and $95 \%$ air. The cells were seeded into 90-mm diameter dishes $\left(20 \times 10^{4} / \mathrm{dish}\right)$ for Western blot analysis or $35-\mathrm{mm}$ diameter glass-bottom dishes $\left(3 \times 10^{4} / \mathrm{dish}\right)$ for immunofluorescence microscopy study. After 5 days, the medium was exchanged to $\alpha$-MEM containing $0.3 \%$ FCS. The cells were then used for experiments after $48 \mathrm{~h}$. When indicated, the cells were pretreated with EGCG at the indicated concentrations.

Western blot analysis. The cultured cells were stimulated by sphingosine 1-phosphate in serum-free $\alpha$-MEM for the indicated periods. The cells were washed twice with phosphatebuffered saline and then lysed, homogenized, sonicated and immediately boiled in a lysis buffer containing $62.5 \mathrm{mM}$ Tris/Cl, $\mathrm{pH}$ 6.8, 2\% sodium dodecyl sulfate (SDS), $50 \mathrm{mM}$ dithiothreitol, and $10 \%$ glycerol. The sample was used for 


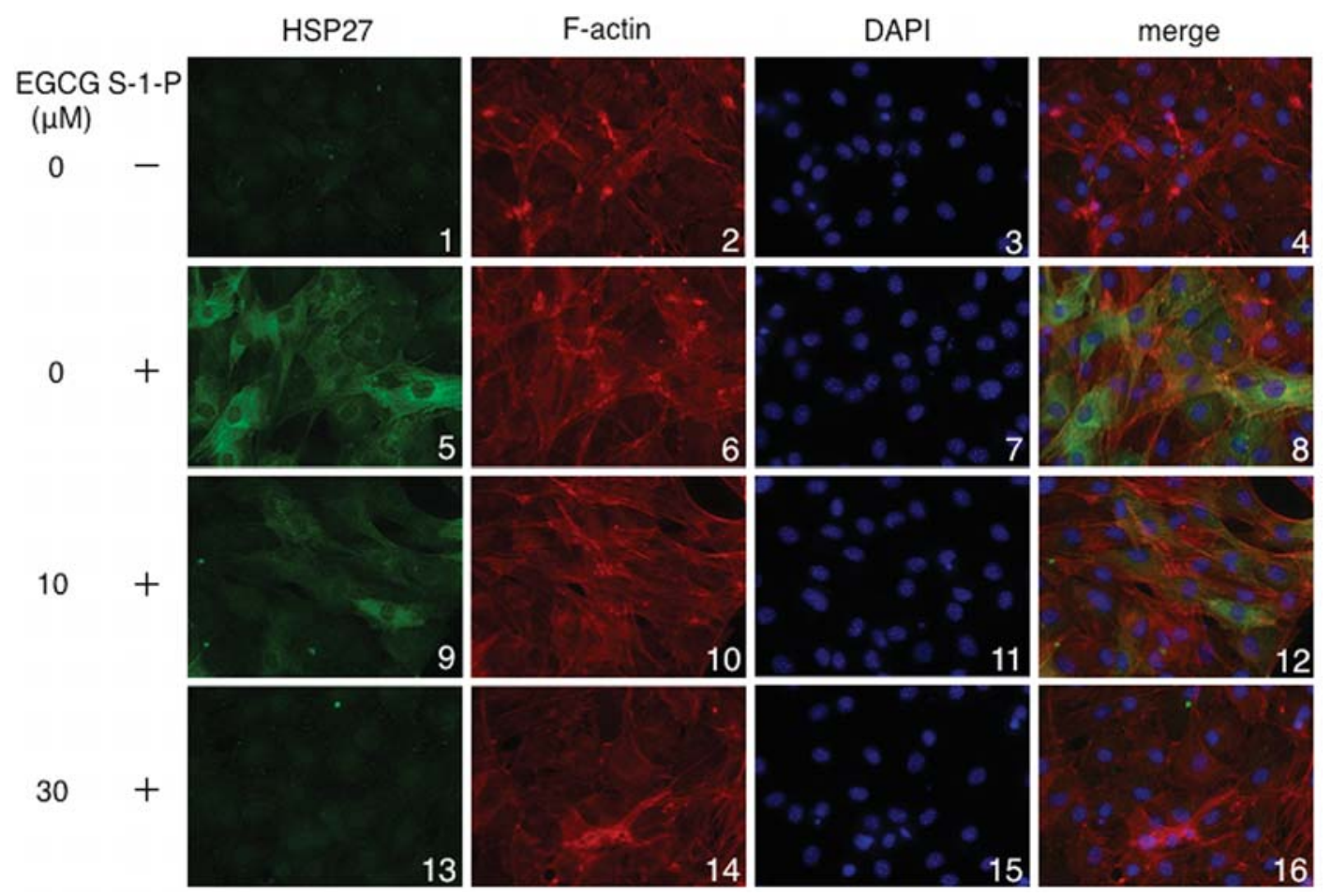

Figure 2. Immunofluorescent microscopy studies of the expression of HSP27. Cells were pretreated with various doses of EGCG for 60 min, and then stimulated with $30 \mu \mathrm{M}$ of sphingosine 1-phosphate for $9 \mathrm{~h}$. The extracts of cells were fixed with paraformaldehyde. After permeabilization of the cells with $0.1 \%$ Triton X-100, cells were exposed to HSP27 antibodies (1:100 dilution), and then treated with Alexa Fluor $488^{\circledR}$ conjugated anti-goat secondary antibodies (green signal). They were then exposed to Alexa Fluor $555^{\circledR}$ phalloidin for F-actin (red signal) and DAPI for nucleus (blue signal) for 20 min and examined by confocal microscopy.

analysis by Western blotting. SDS-polyacrylamide gel electrophoresis (PAGE) was performed by the method of Laemmli (26) in $10 \%$ polyacrylamide gel. Western blot analysis was performed as described previously (27), using antibodies for HSP27, GAPDH, phospho-specific p38 MAP kinase, p38 MAP kinase, phospho-specific MAPKAP-2, MAPKAP-2, phospho-specific Akt, Akt, phospho-specific GSK-3ß or GSK-3ß with peroxidase-labeled antibodies raised in goat anti-rabbit IgG which were used as second antibodies. Peroxidase activity on PVDF membranes was visualized on $\mathrm{X}$-ray film by means of the ECL Western blotting detection system and was quantitated using NIH image software. All of Western blot analyses were repeated at least three times in independent experiments.

Immunofluorescence microscopy study. The cultured cells were pretreated with EGCG at the indicated concentrations for $1 \mathrm{~h}$ and then exposed to sphingosine 1-phosphate $(30 \mu \mathrm{M})$ for $9 \mathrm{~h}$. They were then fixed with $3 \%$ paraformaldehyde for $10 \mathrm{~min}$ on ice and exposed to $0.1 \%$ Triton $\mathrm{X}-100$ for $10 \mathrm{~min}$ to permeabilize the cell membrane, and they were then exposed to HSP27 antibodies (1:100 dilution) in the presence of $1 \%$ BSA for $1 \mathrm{~h}$, followed by exposure to Alexa Fluor $488^{\circledR}$ conjugated calf anti-goat IgG antibodies (1:500) for $1 \mathrm{~h}$. Finally, they were exposed to Alexa Fluor $555^{\circledR}$ phalloidin and DAPI for $20 \mathrm{~min}$. The cells were examined by fluorescent microscopy, BIOREVO (BZ-9000) (Keyence, Tokyo, Japan) following manufacturer's protocol.

Statistical analysis. The data were analyzed by ANOVA followed by Bonferroni method for multiple comparisons between pairs, and $\mathrm{p}<0.05$ was considered significant. All data are presented as the mean $\pm \mathrm{SEM}$ of triplicate determinations.

\section{Results}

Effect of EGCG on the induction of HSP27 stimulated by sphingosine 1-phosphate in MC3T3-E1 cells. We previously showed that sphingosine 1-phosphate stimulated the induction of HSP27 via p38 MAPK and PI3-kinase/Akt in osteoblastlike MC3T3-E1 cells $(16,17)$. Since it was reported that catechin, including EGCG, suppresses bone resorption $(20,21)$, it is of interest to examine the effect of EGCG on the induction of HSP27 stimulated by sphingosine 1-phosphate in these cells. The cells were pretreated with EGCG and then exposed to sphingosine 1-phosphate and we measured expression levels of HSP27 by Western blot analysis (Fig. 1). As a result, EGCG suppressed the induction of HSP27 stimulated by sphingosine 1-phosphate in a dose-dependent manner between 10 and $30 \mu \mathrm{M}$.

We next examined the effect of EGCG on the induction of HSP27 stimulated by sphingosine 1-phosphate using immunofluorescence microscopy (Fig. 2). As shown in Fig. 2, we also found that sphingosine 1-phosphate certainly stimulated the induction of HSP27 (green signal) in the cytosol of these cells (Fig. 2; panels 1 and 5), consistent with the results shown in Fig. 1. EGCG $(10 \mu \mathrm{M})$ clearly suppressed the induction of HSP27 stimulated by sphingosine 1-phosphate (Fig. 2; panel 9) and $30 \mu \mathrm{M}$ of EGCG almost completely suppressed the induction of HSP27 (Fig. 2; panel 13), also consistent with the results shown in Fig. 1. In addition, we examined the effect of EGCG on actin polymerization using phalloidin which reveals F-actin (red signal), since HSP27 is well known to be 
A
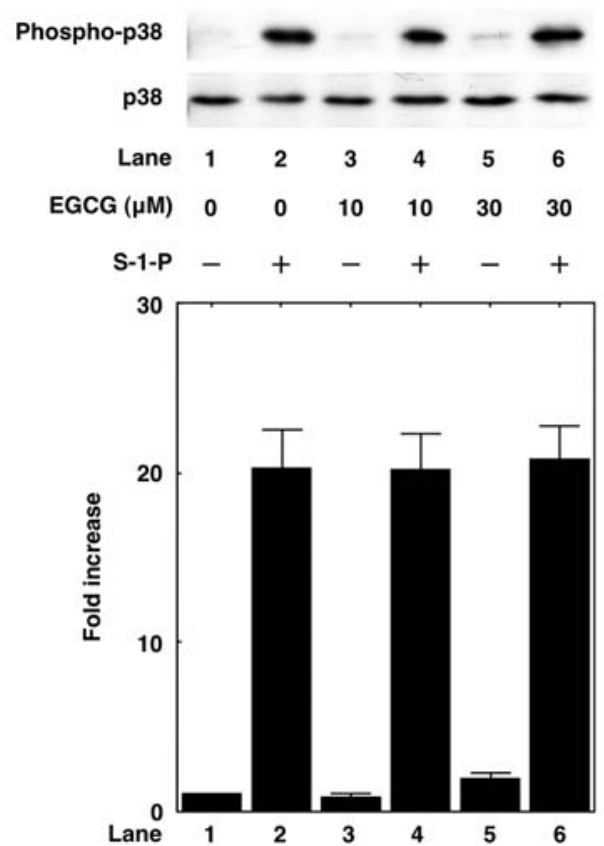

B
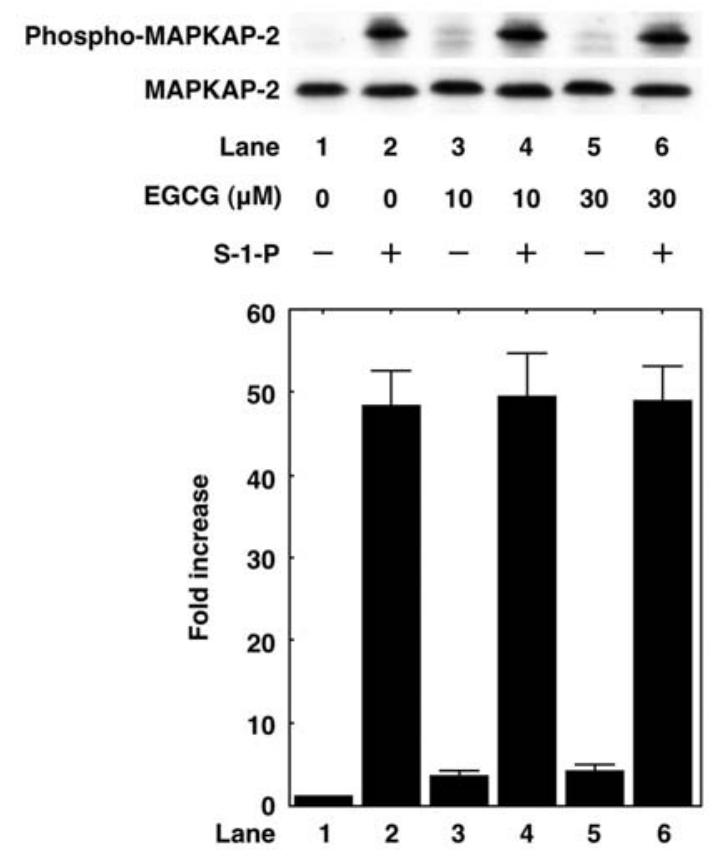

Figure 3. Effect of EGCG on p38 MAP kinase pathway in MC3T3-E1 cells. The cultured cells were pretreated with various doses of EGCG for 60 min, and then stimulated with $30 \mu \mathrm{M}$ of sphingosine 1-phosphate or vehicle for $10 \mathrm{~min}$. The extracts of cells were subjected to SDS-PAGE with subsequent Western blot analysis with antibodies against (A), phospho-specific p38 MAP kinase/p38 MAP kinase or (B), phospho-specific MAPKAP-2/MAPKAP-2. The lower histogram shows quantitative representations of the levels of sphingosine 1-phosphate-stimulated phosphorylation obtained from laser densitometric analysis of three independent experiments, respectively. Each value represents the mean \pm SEM of triplicate determinations. Similar results were obtained with two additional and different cell preparations.

associated with cytoskeleton (28). However, we did not find any significant differences in actin polymerization (Fig. 2; panels 2, 6, 10 and 14).

Effects of EGCG on phosphorylation of p38 MAP kinase and MAPKAP-2 stimulated by sphingosine 1-phosphate in MC3T3-E1 cells. In our previous study (16), we showed that activation of p38 MAP kinase mediates, at least in part, the induction of HSP27 by sphingosine 1-phosphate in osteoblastlike MC3T3-E1 cells. Therefore, we next examined the effect of EGCG on the phosphorylation of p38 MAP kinase stimulated by sphingosine 1-phosphate. However, EGCG did not influence the phosphorylation of p38 MAP kinase in MC3T3-E1 cells even if the cells were pretreated with $30 \mu \mathrm{M}$ of EGCG (Fig. 3A). We also examined the effect of an extremely high dose of EGCG $(100 \mu \mathrm{M})$ on the phosphorylation of MAPKAP-2 and we did not find any significant attenuation of them (data not shown).

Since it is well known that MAPKAP-2 is one of the p38 MAP kinase substrates in the HSP27 phosphorylation $(2,29)$, we next examined the effect of EGCG on the phosphorylation of MAPKAP-2. As a result, sphingosine 1-phosphate stimulated the phosphorylation of MAPKAP-2 in a timedependent manner (data not shown). EGCG did not influence the phosphorylation of MAPKAP-2 in these cells even if the cells were pretreated with $30 \mu \mathrm{M}$ of EGCG (Fig. 3B). We also examined the effect of an extremely high dose of EGCG $(100 \mu \mathrm{M})$ on the phosphorylation of MAPKAP-2 and we did not find any significant attenuation of them (data not shown).

Effect of EGCG on PI3-kinase/Akt pathway in MC3T3-E1 cells. We previously reported that PI3-kinase/Akt pathway, in addition to p38 MAP kinase pathway, plays a part in the process of HSP27 induction stimulated by sphingosine 1-phosphate in osteoblast-like MC3T3-E1 cells $(16,17)$. In order to clarify whether Akt is involved in the suppressive effect of EGCG on the induction of HSP27 in these cells, we next examined the effect of EGCG on the phosphorylation of Akt stimulated by sphingosine 1-phosphate. As shown in Fig. 4A, $30 \mu \mathrm{M}$ of EGCG markedly reduced the phosphorylation of Akt stimulated by sphingosine 1-phosphate.

Since it is well known that GSK-3ß is one of the Akt substrates (30), we further examined the effect of EGCG on their phosphorylation (Fig. 4B). We found that sphingosine 1-phosphate stimulated the phosphorylation of GSK-3B in a time-dependent manner (data not shown). EGCG significantly reduced the phosphorylation of GSK-3ß when the cells were pretreated with $30 \mu \mathrm{M}$ of EGCG (Fig. 4B).

\section{Discussion}

In the present study, we first demonstrated that EGCG significantly suppressed the induction of HSP27 stimulated by sphingosine 1-phosphate in osteoblast-like MC3T3-E1 cells (Fig. 1). Moreover, in immunofluorescence microscopy studies, we also showed that EGCG suppressed the induction of HSP27 stimulated by sphingosine 1-phosphate in a dosedependent manner (Fig. 2). It is well recognized that the MAP kinase superfamily plays an important role in cellular functions including proliferation, differentiation, and cell death in a variety of cells (29). Three major MAP kinases such as p44/p42 MAP kinase, p38 MAP kinase and SAPK/JNK are known as central elements used by mammalian cells to transduce the diverse messages. We previously showed that p38 MAP kinase 
A

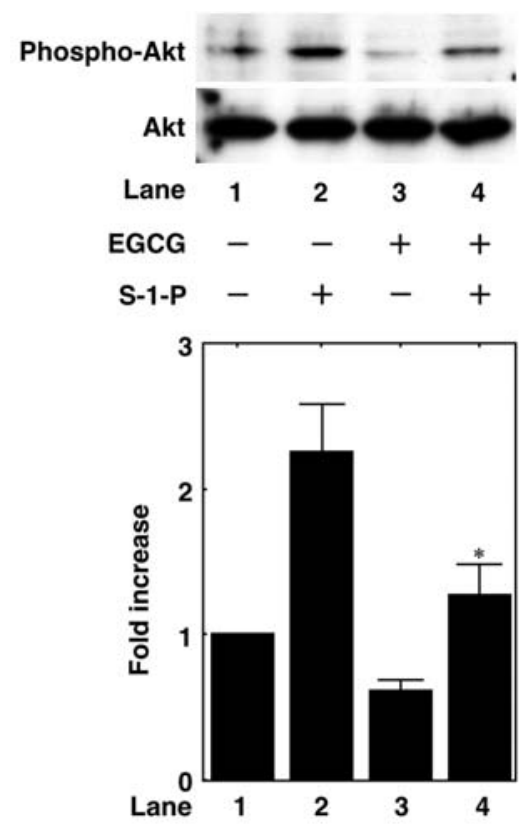

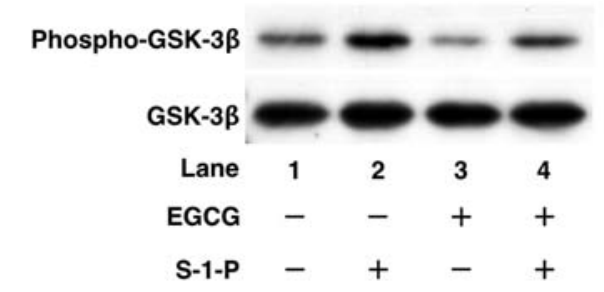

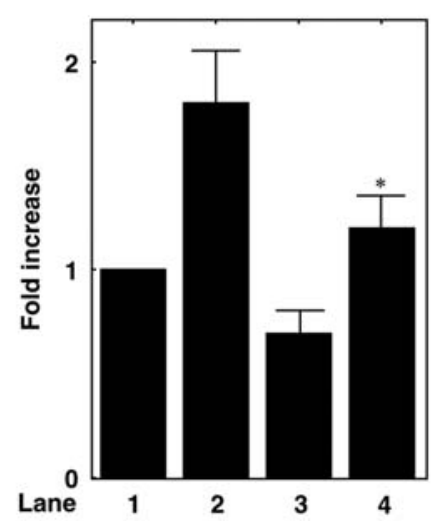

Figure 4. Effect of EGCG on PI-3 kinase/Akt pathway in MC3T3-E1 cells. The cultured cells were pretreated with $30 \mu \mathrm{M}$ of EGCG for 60 min, and then stimulated with $30 \mu \mathrm{M}$ of sphingosine 1-phosphate or vehicle for $15 \mathrm{~min}$. The extracts of cells were subjected to SDS-PAGE with subsequent Western blotting with antibodies against (A), phospho-specific Akt/Akt or (B), phospho-specific GSK-3ß/GSK-3ß. The lower histogram shows quantitative representations of the levels of sphingosine 1-phosphate-stimulated phosphorylation obtained from laser densitometric analysis of three independent experiments, respectively. Each value represents the mean \pm SEM of triplicate determinations. Similar results were obtained with two additional and different cell preparations. The asterisks indicate a significant decrease $\left({ }^{*} \mathrm{p}<0.05\right)$ with respect to the control (lane 2 , respectively).

among the MAP kinase superfamily functions as a positive regulation in the induction of HSP27 induced by sphingosine 1-phosphate in osteoblast-like MC3T3-E1 cells (16). In the present study, phosphorylation of p38 MAP kinase stimulated by sphingosine 1-phosphate was not affected by EGCG (Fig. 3A). Additionally, EGCG had little effect on phosphorylation of MAPKAP-2 stimulated by sphingosine 1-phosphate (Fig. 3B), a well known substrate of p38 MAP kinase (29). Based on our findings, it seems unlikely that the suppressive effect of EGCG on the induction of HSP27 stimulated by sphingosine 1-phosphate is due to the inhibition of p38 MAP kinase pathway in osteoblast-like MC3T3-E1 cells.

We recently reported that sphingosine 1-phosphate stimulates the HSP27 induction via PI3-kinase/Akt pathway independently of p38 MAP kinase pathway in osteoblast-like MC3T3-E1 cells (17). Therefore, we next examined the effect of EGCG on HSP27 levels and PI3-kinase/Akt pathway in these cells. We found that sphingosine 1-phosphate-induced phosphorylation of Akt was significantly attenuated by EGCG (Fig. 4A). In addition, EGCG also suppressed the phosphorylation of GSK-3ß, well known as a substrate of Akt (30), stimulated by sphingosine 1-phosphate (Fig. 4B). Taking our findings into account, it is probable that the inhibitory effect of EGCG on the induction of HSP27 stimulated by sphingosine 1-phosphate is closely related to the suppression of PI3-kinase/Akt pathway in osteoblast-like MC3T3-E1 cells. The potential mechanism is summarized in Fig. 5. We recently reported that EGCG inhibits prostaglandin $\mathrm{D}_{2}$-stimulated HSP27 induction via the suppression of p44/p42 MAP kinase pathway in osteoblast-like MC3T3-E1 cells (23). However, we confirmed that p44/p42 MAP kinase does not take part in the induction of HSP27 stimulated by

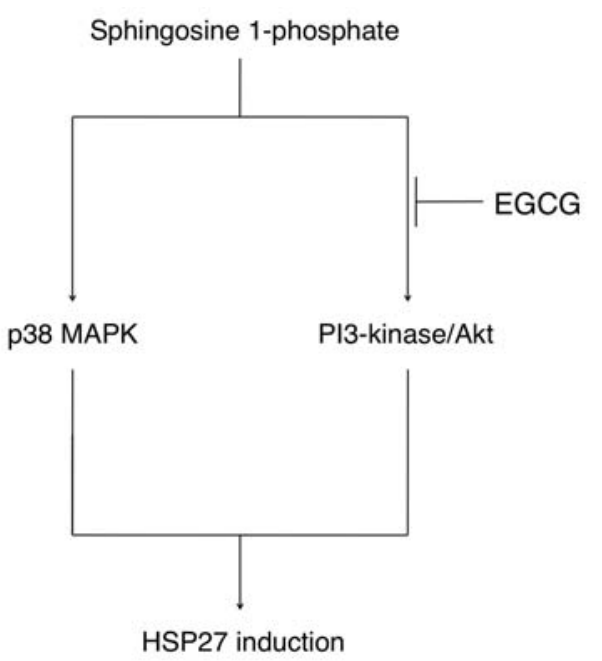

Figure 5. Diagram of the potential mechanism of the suppression by EGCG of the sphingosine 1-phosphate-stimulated HSP27 induction in MC3T3-E1 cells. EGCG, (-)-epigallocatechin gallate; p38 MAPK, p38 mitogenactivated protein kinase; PI3-kinase, phosphatidylinositol 3-kinase; HSP27, heat shock protein 27 .

sphingosine 1-phosphate in these cells (16). Thus, it is possible that EGCG affects several parts of signaling involved in HSP27 induction in osteoblasts. Further investigations are necessary to elucidate the detail mechanism behind the down-regulation by EGCG of HSP27 induction in osteoblasts.

We recently reported that phosphorylated HSP27 represses growth of hepatocellular carcinoma via inhibition of extracellular-signal-regulated kinase (31). Although the physiological significance of HSP27 in osteoblasts is not yet 
clarified, one possibility is that HSP27 induced by sphingosine 1-phosphate, which is repressed by the treatment with EGCG, suppresses cell proliferation in osteoblasts. As for bone metabolism, it is currently recognized that sphingosine 1-phosphate produced by osteoclasts plays a pivotal role in the recruitment of osteoprogenitors to bone resorption sites through promoting the migration and the survival osteoblasts (14). In addition, it was shown that catechin has an inhibitory effect on bone resorption (20). Consistent with this, catechin reportedly induces apoptotic cell death of osteoclasts (32). Moreover, it was reported that catechin increases cell viability and alkaline phosphatase activity in osteoblast-like MC3T3-E1 cells and apoptosis of these cells is suppressed by catechin (21). This evidence leads us to speculate that the inhibitory effect of EGCG on the induction of HSP27 promotes bone formation and reduces bone resorption, resulting in at least preventing bone loss. Based on these findings, intake of catechin-containing beverage, such as green tea, prevents the progression of postmenopausal osteoporosis. It is probable that EGCGinduced suppression of the PI3-kinase/Akt cascade in osteoblasts contributes to the modulation of osteoblastic cell function toward bone formation, at least in part, via specifically down regulating HSP27 induction. Our present findings provide new insight into pharmacological effects of catechin action on osteoblasts. Further investigations are necessary to clarify the exact mechanism underlying the favorable effects of catechin on bone metabolism.

In conclusion, our present results strongly suggest that EGCG suppresses the induction of HSP27 stimulated by sphingosine 1-phosphate via suppression of PI3-kinase/Akt pathway in osteoblasts.

\section{Acknowledgements}

We are very grateful to Yoko Kawamura for her skillful technical assistance. This work was supported in part by a Grant-in-Aid for Scientific Research (16590873 and 16591482) from the Ministry of Education, Science, Sports and Culture of Japan, the Research Grants for Longevity Sciences (15A-1 and 15C-2), Research on Proteomics and Research on Fracture and Dementia from Ministry of Health, Labour and Welfare of Japan.

\section{References}

1. Hendrick JP and Hartl FU: Molecular chaperone functions of heat-shock proteins. Annu Rev Biochem 62: 349-384, 1993.

2. Benjamin IJ and McMillan DR: Stress (heat shock) proteins: molecular chaperones in cardiovascular biology and disease. Circ Res 83: 117-132, 1998.

3. Inaguma Y, Goto S, Shinohara H, Hasegawa K, Ohshima K and Kato K: Physiological and pathological changes in levels of the two small stress proteins, HSP27 and $\alpha B$ crystallin, in rat hindlimb muscles. J Biochem 114: 378-384, 1993.

4. Gaestel M, Schroder W, Benndorf R, Lippmann C, Buchner K, Hucho F, Erdmann VA and Bielka H: Identification of the phosphorylation sites of the murine small heat shock protein hsp25. J Biol Chem 266: 14721-14724, 1991.

5. Landry J, Lambert H, Zhou M, Lavoie JN, Hickey E, Weber LA and Anderson CW: Human HSP27 is phosphorylated at serines 78 and 82 by heat shock and mitogen-activated kinases that recognize the same amino acid motif as S6 kinase II. J Biol Chem 267: 794-803, 1992.

6. Kato K, Hasegawa K, Goto S and Inaguma Y: Dissociation as a result of phosphorylation of an aggregated form of the small stress protein, hsp27. J Biol Chem 269: 11274-11278, 1994.
7. Rogalla T, Ehrnsperger M, Preville X, Kotlyarov A, Lutsch G, Ducasse C, Paul C, Wieske M, Arrigo AP, Buchner J and Gaestel M: Regulation of Hsp27 oligomerization, chaperone function, and protective activity against oxidative stress/tumor necrosis factor $\alpha$ by phosphorylation. J Biol Chem 274: 18947-18956, 1999

8. Nijweide PJ, Burger EH and Feyen JH: Cells of bone: proliferation, differentiation, and hormonal regulation. Physiol Rev 66: 855-886, 1986.

9. Shakoori AR, Oberdorf AM, Owen TA, Weber LA, Hickey E, Stein JL, Lian JB and Stein GS: Expression of heat shock genes during differentiation of mammalian osteoblasts and promyelocytic leukemia cells. J Cell Biochem 48: 277-287, 1992.

10. Cooper LF and Uoshima K: Differential estrogenic regulation of small M(r) heat shock protein expression in osteoblasts. J Biol Chem 269: 7869-7873, 1994.

11. Sanchez T and Hla T: Structural and functional characteristics of S1P receptors. J Cell Biochem 92: 913-922, 2004.

12. Spiegel S and Merrill AH Jr: Sphingolipid metabolism and cell growth regulation. FASEB J 10: 1388-1397, 1996.

13. Spiegel S and Milstien S: Sphingosine-1-phosphate: an enigmatic signalling lipid. Nat Rev Mol Cell Biol 4: 397-407, 2003.

14. Khosla S, Westendorf JJ and Oursler MJ: Building bone to reverse osteoporosis and repair fractures. J Clin Invest 118: 421-428, 2008

15. Grey A, Chen Q, Callon K, Xu X, Reid IR and Cornish J: The phospholipids sphingosine-1-phosphate and lysophosphatidic acid prevent apoptosis in osteoblastic cells via a signaling pathway involving G(i) proteins and phosphatidylinositol-3 kinase. Endocrinology 143: 4755-4763, 2002.

16. Kozawa O, Niwa M, Matsuno H, Tokuda H, Miwa M, Ito H, Kato $\mathrm{K}$ and Uematsu T: Sphingosine 1-phosphate induces heat shock protein 27 via p38 mitogen-activated protein kinase activation in osteoblasts. J Bone Miner Res 14: 1761-1767, 1999.

17. Takai S, Tokuda H, Matsushima-Nishiwaki R, Hanai Y, Kato K and Kozawa O: Phosphatidylinositol 3-kinase/Akt plays a role in sphingosine 1-phosphate-stimulated HSP27 induction in osteoblasts. J Cell Biochem 98: 1249-1256, 2006.

18. Harborne JB and Williams CA: Advances in flavonoid research since 1992. Phytochemistry 55: 481-504, 2000.

19. Jankun J, Selman SH, Swiercz R and Skrzypczak-Jankun E: Why drinking green tea could prevent cancer. Nature 387: 561, 1997.

20. Delaisse JM, Eeckhout Y and Vaes G: Inhibition of bone resorption in culture by $(+)$-catechin. Biochem Pharmacol 35: 3091-3094, 1986.

21. Choi EM and Hwang JK: Effects of (+)-catechin on the function of osteoblastic cells. Biol Pharm Bull 26: 523-526, 2003.

22. Tokuda H, Takai S, Matsushima-Nishiwaki R, Akamatsu S, Hanai Y, Hosoi T, Harada A, Ohta T and Kozawa O: (-)-Epigallocatechin gallate enhances prostaglandin $\mathrm{F}_{2_{\alpha}}$-induced VEGF synthesis via upregulating SAPK/JNK activation in osteoblasts. J Cell Biochem 100: 1146-1153, 2007.

23. Yamauchi J, Takai S, Matsushima-Nishiwaki R, Hanai Y, Doi T, Kato H, Ogura S, Kato K, Tokuda H and Kozawa O: (-)Epigallocatechin gallate inhibits prostaglandin $\mathrm{D}_{2}$-stimulated HSP27 induction via suppression of the p44/p42 MAP kinase pathway in osteoblasts. Prostaglandins Leukot Essent Fatty Acids 77: 173-179, 2007.

24. Sudo H, Kodama HA, Amagai Y, Yamamoto S and Kasai S: In vitro differentiation and calcification in a new clonal osteogenic cell line derived from newborn mouse calvaria. J Cell Biol 96: 191-198, 1983.

25. Kozawa O, Suzuki A, Tokuda $\mathrm{H}$ and Uematsu T: Prostaglandin $\mathrm{F}_{2}$ stimulates interleukin-6 synthesis via activation of PKC in osteoblast-like cells. Am J Physiol 272: E208-E211, 1997.

26. Laemmli UK: Cleavage of structural proteins during the assembly of the head of bacteriophage T4. Nature 227: 680-685, 1970.

27. Kato K, Ito H, Hasegawa K, Inaguma $\mathrm{Y}$, Kozawa $\mathrm{O}$ and Asano T: Modulation of the stress-induced synthesis of hsp27 and $\alpha \mathrm{B}$-crystallin by cyclic AMP in C6 rat glioma cells. J Neurochem 66: 946-950, 1996.

28. Zhu Y, O'Neill S, Saklatvala J, Tassi L and Mendelsohn ME: Phosphorylated HSP27 associates with the activation-dependent cytoskeleton in human platelets. Blood 84: 3715-3723, 1994

29. Widmann C, Gibson S, Jarpe MB and Johnson GL: Mitogenactivated protein kinase: conservation of a three-kinase module from yeast to human. Physiol Rev 79: 143-180, 1999. 
30. Cross DA, Alessi DR, Cohen P, Andjelkovich M and Hemmings BA: Inhibition of glycogen synthase kinase- 3 by insulin mediated by protein kinase B. Nature 378: 785-789, 1995.

31. Matsushima-Nishiwaki R, Takai S, Adachi S, Minamitani C, Yasuda E, Noda T, Kato K, Toyoda H, Kaneoka Y, Yamaguchi A, Kumada T and Kozawa O: Phosphorylated heat shock protein 27 represses growth of hepatocellular carcinoma via inhibition of extracellular signal-regulated kinase. J Biol Chem 283: 1885218860, 2008.
32. Nakagawa H, Wachi M, Woo JT, Kato M, Kasai S, Takahashi F, Lee IS and Nagai K: Fenton reaction is primarily involved in a mechanism of (-)-epigallocatechin-3-gallate to induce osteoclastic cell death. Biochem Biophys Res Commun 292: 94-101, 2002. 